\title{
Design of Detecting System for Dust Concentration in Flour Processing Factory Based on SCM
}

\author{
Caiyun Gui ${ }^{1}$ and Baobao Kang ${ }^{2}$ \\ ${ }^{1}$ Yulin University, Yulin 719000 \\ ²BGP INC.,CHINA NATIONAL PETROLEUM CORPORATION, zhuozhou, 071751
}

Keywords: Dust concentration; Oxygen concentration; Detecting system design; Simulation

\begin{abstract}
The dust concentration of flour processing factory ifthe control is not good, it will not only improve the incidence rate of workers occupation disease, but also can easily cause dust explosion prevention, in order to control the harm, the system uses STC89C52 as the main control chip, combined with the dust concentration sensor and the oxygen concentration sensor, A/D converter, LCD display circuit and the module of warning circuit etc.to realize the dust concentration and oxygen concentration value of the real-time detection and display, and in a safe range automatically give an alarm signal. The simulation shows that the system can monitor the concentration of oxygen and dust in a relatively confined space in real time. The system is simple and easy to operate, and has certain practical value.
\end{abstract}

\section{Introduction}

Since the first dust explosion in Italy's durin flour mill in 1785 , there have been many serious dust explosions in the world. Studies have shown that the combustion heat and maximum explosion pressure of grain dust are even higher than that of explosives and glycerol. This kind of harm is huge. Although scientists in various countries have conducted a lot of discussions and research on the prevention and protection of dust explosions, dust explosions have so far been one of the most important sources of threats to safe industrial production in modern processing, transportation and storage. The main conditions for dust explosion to meet are: combustible dust; sufficient oxygen; ignition source or the mutual friction and vibration of dust; dust suspended into a certain concentration of dust clouds. The surface of the dust adsorbs oxygen in the air, and the greater the concentration is, the more oxygen is absorbed and the static electricity is more easily carried, and the explosion is more likely to occur. Most of the domestic flour processing factories adopt wind net design to prevent the formation of dust clouds, but the location where the wind cannot reach is a potential place for danger. Real-time monitoring of risk factors at "blind spots" can effectively prevent accidents.

The design takes STC89C52 single-chip microcomputer as the controller, uses DSM501A dust concentration sensor, oxygen concentration sensor, A/D converter, buzzer and other systems to realize the detection of oxygen concentration and dust concentration, which will be displayed on LCD display after processing by single-chip microcomputer. The hardware circuit is drawn by Altium Designer and written in $\mathrm{C}$ language program. The simulation is done in Proteus software environment.

\section{System Overall Design Scheme and Hardware Circuit}

Overall Structural Design. The overall system consists of a central processing circuit, a sensor signal detection circuit, a signal processing circuit, a minimum system of a single-chip microcomputer, a keyboard circuit for input, an acousto-optic alarm for output and an LCD display circuit. The diagram is shown in Fig. 1. 


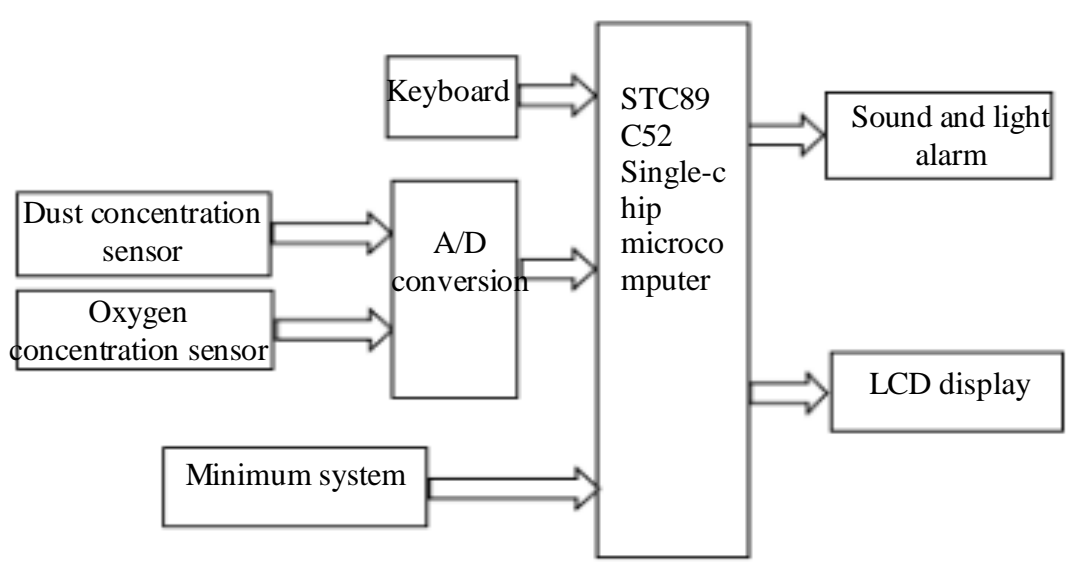

Figure 1. Finite Diagram of hardware

Dust Sensor and Circuit Design. DSM501A is a dust sensor produced by SYHITECH of South Korea. It consists of a light source, a light detector and a heater.The sensor uses the principle of particle counting, and the built-in heater enables the air in the detection environment to be automatically drawn into the sensor. After the dust particles are irradiated by the detection light emitted by the light source, the probe light is scattered, and the scattered light is converged by lens and converted by photoelectricity. The device converts the optical signal into an electrical signal and finally converts it into DC pulse signal and output. Therefore, when the dust sensor is used, no A/D conversion is required. The detection circuit is shown in Fig. 2 .

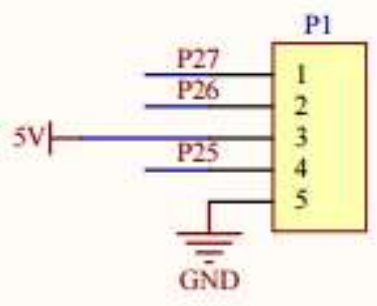

Figure 2. Finite Dust detection circuit

Oxygen Sensor and Circuit Design. The oxygen sensor adopts O2-A2. Its working principle is based on an electrochemical galvanic cell. The gas to be measured is generated in the primary battery, the oxidation of the anode and the reduction process of the cathode generate electric current. During the electrochemical reaction, the current generated of the gas to be measured is proportional to its concentration and the oxygen concentration is calculated. ADC0832 only recognizes the voltage signal, so the output current signal is converted into the output voltage signal. The detection circuit is shown in Fig. 3.

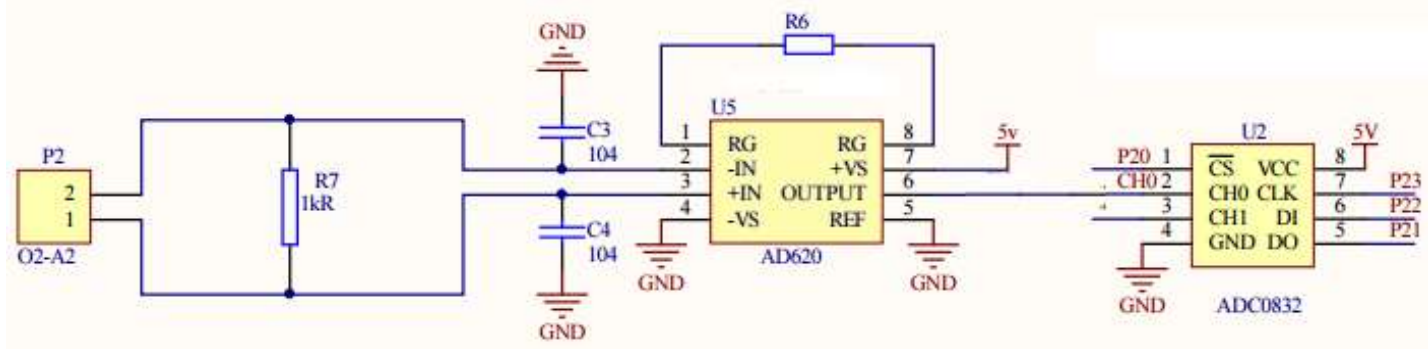

Figure 3. Finite Oxygen detection circuit

Selection of Display Methods. LCD is a passive display device with extremely low power consumption and is widely used in portable instrumentation or low-power display devices. The 
character LCD is designed to display symbols, such as numbers, letters and graphics. The dust concentration and oxygen concentration threshold can be displayed at the same time in the design. Therefore, LCD displays with 2 or more lines must be selected.

Alarm Circuit Design. Taking into account the practical value and cost, buzzer and LED light emitting alarm are used. When the dust concentration reaches the set safety threshold, the buzzer will sound and the red LED lights will be on. When the oxygen concentration reaches the set safety threshold, the buzzer will sound and yellow lights will be on, so that sound alarms and light alarms are achieved.

\section{Programming}

The program design is carried out according to the modularization, and consists of two main modules: the main program and the subroutine. The subroutines also contain keys, D/A conversion and display. The main program is shown in Fig. 4.

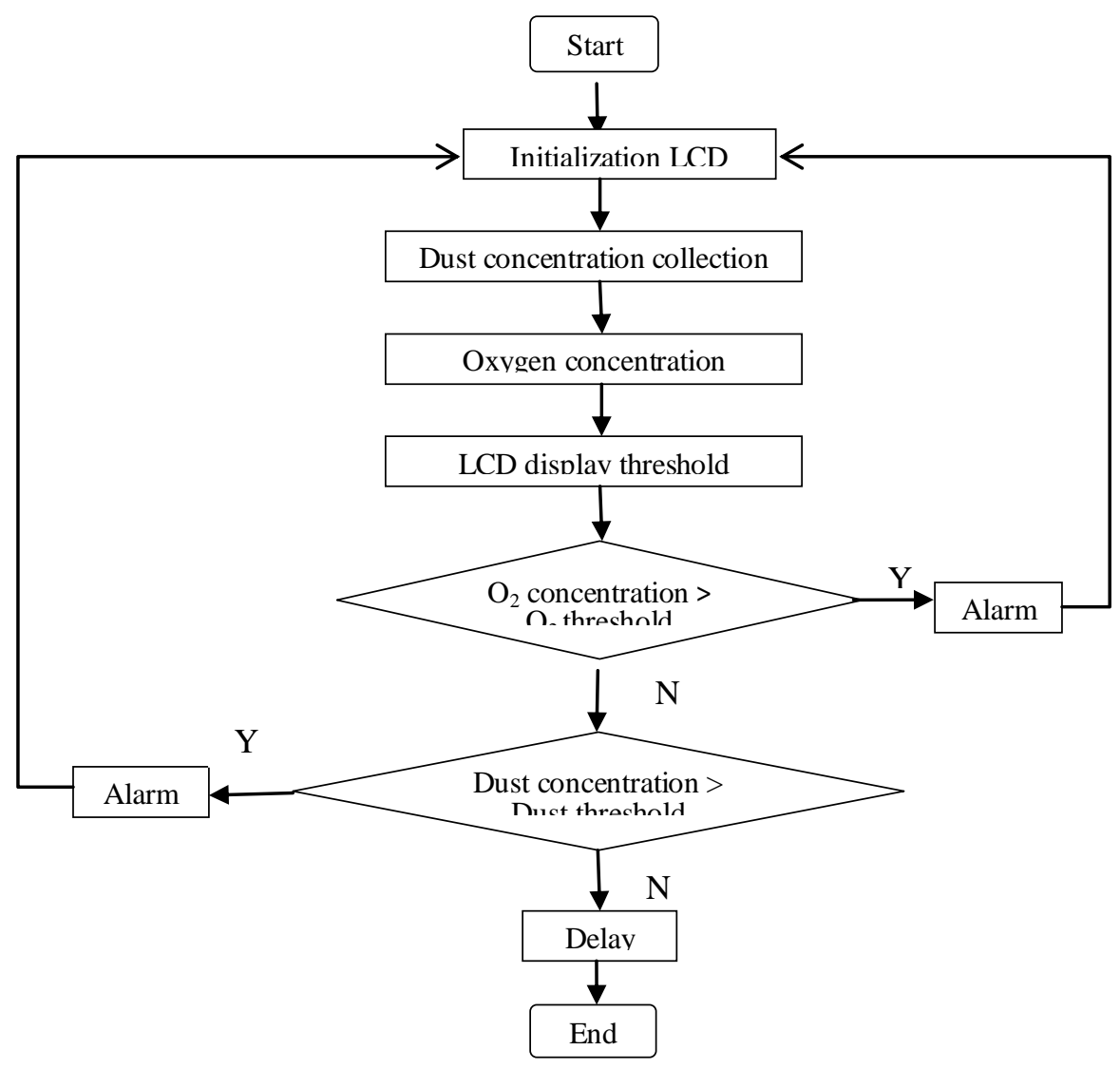

Figure 4. Finite Main program flow chart

\section{Simulation}

Use Proteus software to perform the entire simulation work, the main simulation is the oxygen concentration, dust concentration settings and alarm indications.

When dust concentration and oxygen concentration reach or exceed their respective maximum thresholds at the same time, the red and yellow lights are on at the same time, and the buzzer alarms, as shown in Fig. 5. 


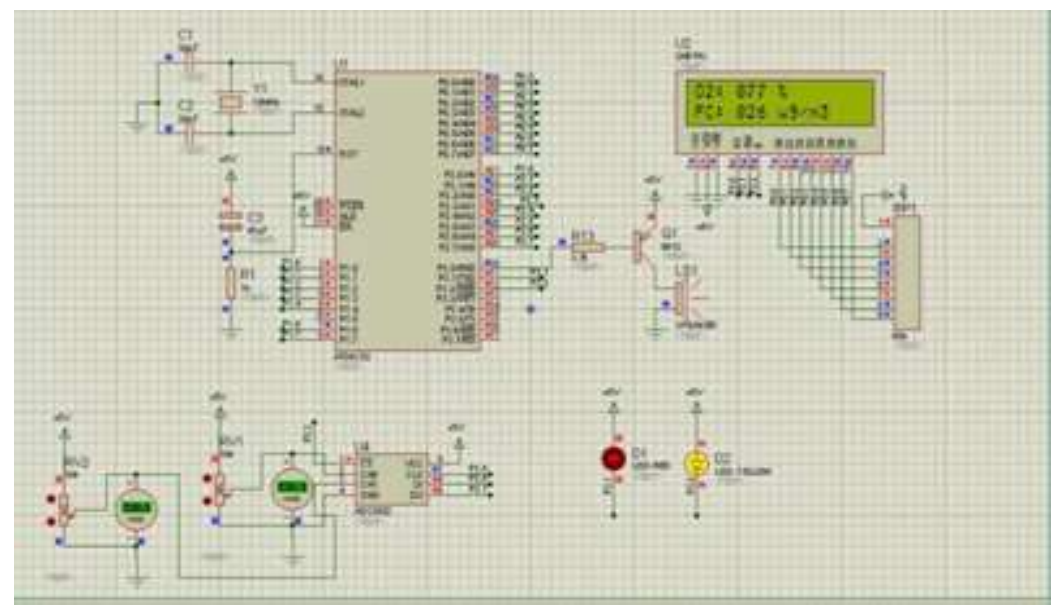

Figure 5. Finite Simulation of dust and oxygen being over the limit at the same time

\section{Conclusion}

This paper designed a detecting system based on STC89C52 single-chip microcomputer for dust concentration and air oxygen content in flour processing factories, which can monitor the internal oxygen concentration and dust concentration of the factory in real time, and promptly reminds the staff of possible unsafe hidden dangers in the form of sound and light alarms to take corresponding measures to prevent accidents, thus ensuring the safety of lives in real time and improving the efficiency of industrial production. Grasp the concentration of dust in the workshop, protect the degree of harm to the human body according to the concentration to prevent the harm of lung diseases, which has a great practical value.

\section{References}

[1] Tang Juan. Status and Development Trend of Dust Concentration On-line Monitoring Technology[J]. Mining Safety and Environmental Protection, 2009, 36(5): 69-74.

[2]Zhang Chaoguang. Preliminary Discussion on Influencing Factors and Protective Measures of Dust Explosion[J]. Coal Chemical Industry, 2005(2): 9-11.

[3]SU Xiuzhen.Improvement Opinions on Microcomputer Simulation System of DVCC52196 Microcontroller[J]. Journal of Zhangzhou Vocational University,2003(4):105-107.

[4] Liu Renqing. Design of Dust Detector Based on Bluetooth[J]. Communications Technology, 2009(3): 252-254.

[5] Zhang Chengbin. On Dust Explosion of Flour Processing Factories[J].Flour News,2008(4): 52-53.

[6] Liu Yongqin.Xu Chunjia. Explosive Characteristics, Classification and Explosion-proof Measures of Combustible Dust[J]. Explosion-proof Electric Machines, 2006(3):14-18.

[7] Xue Fulian. Dust Explosion and the Protection[J]. Mechanical Engineering and Automation, 2009(3):14-15.

[8] Liu Qi, Tan Yingxin. Basic Characteristics of Dust Explosion and Protection Measures[J]. Industrial Safety and Environmental Protection, 2008, 34(3): 17-18.

[9] Guo Yanfei, Zhou Jianxin, Chen Weihong. Investigation and Analysis of Dust Hazard Events[J]. China Safety Science and Technology, 2009,5(4):80-84.

[10] Jia Yutao. Grain Dust Explosion and Prevention in Flour Enterprises [D]. Shenyang: Northeastern University, 2011. 